\title{
Steady-State Creep of a Long Narrow Membrane inside a High Rigid Matrix at Variable Transverse Pressure
}

\author{
A.M. Lokoshchenko ${ }^{1 *}$, L.V.Fomin ${ }^{1,2 * *}$, Yu.G. Basalov $1 * * *$ \\ ${ }^{1}$ Lomonosov Moscow State University. Research Institute of Mechanics \\ 119192 1, Michurinsky pr., Moscow, Russian Federation \\ *e-mailloko@imec.msu.ru;** e-mail fleonid1975@mail.ru ;*** e-mail basalov@yandex.ru. \\ ${ }^{2}$ Samara State Technical University. Department of Applied Mathematics and Computer Science \\ 443100 144, Molodogvardejskaya st., Samara, Russian Federation \\ fleonid1975@mail.ru
}

\begin{abstract}
The problem of the steady-state creep of a long narrow rectangular membrane with a proportional dependence of the transverse pressure on time is investigated. This membrane is located inside a high long rigid matrix of rectangular cross-section, in which the ratio of the height to half the width is not less than 1. A power relationship between the stress intensities and the creep strain rates of the membrane is used. Two variants of the conditions of contact between the membrane and the matrix are considered - ideal sliding and adhesion. The analysis is carried out until the time of almost complete adherence of the membrane to the matrix. A comparison is made of these times during sliding and adhesion for different values of the exponent in the constitutive equation and the relative height of the matrix.
\end{abstract}

Keywords: membrane, the steady-state creep, a high rigid matrix, variable transverse pressure, ideal slip, adhesion.

\section{Introduction}

Consider the creep of a long narrow rectangular membrane fixed along the long sides and loaded with uniform transverse pressure $q$, which increases in proportion to time $t$. The solution to this problem under various physical and geometric conditions is given in the monographs of L.M. Kachanov [1], Odqvist [2], N.N. Malinin [3] and others. Of particular interest is the study of the creep of the considered membrane inside a rigid matrix. In monographs [3, 4], a cycle of problems on the creep of such a membrane inside a rigid matrix is considered. In [4], solutions of problems are given taking into account various forms of matrices: wedge-shaped, curvilinear and rectangular under various conditions at the contact between the membrane and the matrix.

\section{Problem Statement}

This paper investigates the creep of a long narrow rectangular membrane of thickness $H_{0}$ inside a rigid rectangular matrix (Fig. 1). Membrane width $2 a$ and length $L$ satisfy the inequality $2 a / L<<1$. The ratio of the height of the matrix $b$ to half of its width $a$ satisfies the inequality $b / a \geq 1$.

To describe the deformation of the membrane at $t>0$, a power-law model of the steady-state creep of the material is used

$$
\dot{p}_{u}=\frac{1}{t_{0}}\left(\frac{\sigma_{u}}{\sigma_{0}}\right)^{n}, p_{u}(t=0)=0,
$$

where $\sigma_{u}, \dot{p}_{u}$-stress intensity and creep strain rates intensity, respectively, $\sigma_{0}, t_{0}$ and $n$-constants of a corresponding dimensions. These values are determined as a result of a series of creep tests of several tensile specimens and the subsequent approximation of the dependence of the steady-state creep rate on stress in the form of a power function. 


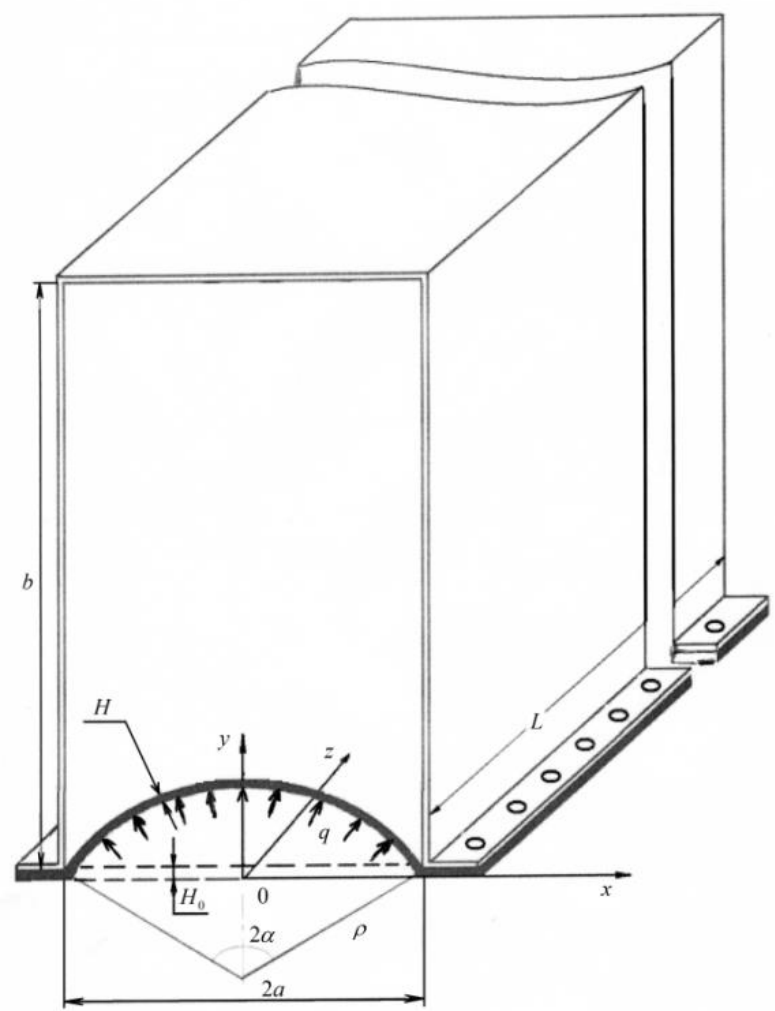

Fig. 1. General view.

Here, the proportional dependence of the magnitude of the transverse pressure $q$ on time $t$ is considered:

$$
q(t)=q_{0} k \frac{t}{t_{0}}=q_{0} k \bar{t}, \bar{t}=\frac{t}{t_{0}}
$$

It is of interest to consider the features of the almost complete adherence of the membrane to the space inside the matrix at different rates of increase of the transverse pressure at which the ratio of the radius of curvature of the middle surface $\rho$ of the membrane to $a$ equals a given small value $\rho / a=\Delta<<1$.

When modeling the stress-strain state at $t>0$, the radial $\sigma_{r r}$, circumferential $\sigma_{\theta \theta}$ and axial $\sigma_{z z}$ principal stresses and the corresponding components of the creep strain tensor $p_{r r}, p_{\theta \theta}$ and $p_{z z}$ are considered.

Considering a membrane element; taking the stresses in the element uniformly distributed over the thickness and writing the equilibrium equations in projections onto the normal and tangent, it is obtained:

$$
\sigma_{\theta \theta}=\frac{q \rho}{H}, \quad d\left(\sigma_{\theta \theta} H\right)=0
$$


where $H$ - membrane thickness.

Therefore,

$$
\sigma_{\theta \theta} H=\text { const. }
$$

Comparing equalities (2) and (3), it is concluded that the middle surface of the membrane during its deformation is a part of the surface of a circular cylinder with an opening angle $2 \alpha$ [4]. Consequently, according to equality

(2), the circumferential stress along the circumference of the radius $\rho$ does not change.

The membrane creep is studied in three successive stages.

\section{Free Deformation of the Membrane under Creep Conditions (The First Stage)}

At this stage, the membrane is deformed under conditions of steady creep until it touches the side walls of the rigid matrix.

Dimensionless variables are introduced as follows:

$$
\begin{aligned}
& \bar{q}=\frac{q}{\sigma_{0}}, \bar{H}_{i}=\frac{H_{i}}{H_{0}}, \bar{H}_{0}=\frac{H_{0}}{a}, \bar{b}=\frac{b}{a}, \bar{q}_{0}=\frac{q_{0}}{\sigma_{0}}, \\
& \bar{t}=\frac{t}{t_{0}}, \quad \bar{\rho}=\frac{\rho}{a}, \bar{\sigma}_{i j}=\frac{\sigma_{i j}}{\sigma_{0}}(i, j=1,2,3),
\end{aligned}
$$

$H_{i}$ - membrane thickness at the $i$-th stage $(i=1 \div 3)$.

Further, the dashes over all dimensionless variables are omitted, and the dot denotes the derivative with respect to the dimensionless time everywhere.

As a connection between the components of stress tensors and creep strain rates, the hypothesis of proportionality of the corresponding deviators is accepted (see, for example, [4]):

$$
\sigma_{u}=\frac{1}{\sqrt{2}} \sqrt{\left(\sigma_{r r}-\sigma_{\theta \theta}\right)^{2}+\left(\sigma_{\theta \theta}-\sigma_{z z}\right)^{2}+\left(\sigma_{z z}-\sigma_{r r}\right)^{2}+6\left(\sigma_{z \theta}^{2}+\sigma_{\theta r}^{2}+\sigma_{z r}^{2}\right)},
$$

$S_{i j}$ - stress deviator components.

In the considered plane deformed state, the rate of axial creep strain $\dot{p}_{z z}$ is taken to be zero:

$$
\dot{p}_{z z}=0 \text {. }
$$

Accept, as usual for thin-walled cylindrical shells, the equality:

$$
\sigma_{r r}=0
$$

In this case, from the creep hypothesis (4), taking into account (5) - (6), it follows: 


$$
\sigma_{z z}=\frac{1}{2} \sigma_{\theta \theta}, \sigma_{u}=\frac{\sqrt{3}}{2} \sigma_{\theta \theta}=\frac{\sqrt{3}}{2} \cdot \frac{q \rho}{H_{0} H_{1}}
$$

Considering two close deformed states of the membrane, the increment of the circumferential creep strain is determined:

$$
d p_{\theta \theta}=\frac{(\rho+d \rho)(\alpha+d \alpha)-\rho \alpha}{\rho \alpha}=\frac{d \rho}{\rho}+\frac{d \alpha}{\alpha} .
$$

Consequently, the circumferential creep strain rate is

$$
\dot{p}_{\theta \theta}=\frac{\dot{\rho}}{\rho}+\frac{\dot{\alpha}}{\alpha}=\left(\frac{1}{\alpha}-\operatorname{ctg} \alpha\right) \dot{\alpha}
$$

From the incompressibility condition in the case of a plane deformed state, it follows:

$$
-\frac{\dot{H}_{1}}{H_{1}}=\left(\frac{1}{\alpha}-\operatorname{ctg} \alpha\right) \dot{\alpha}, \alpha(t=0)=0, H_{1}(t=0)=0, H_{1}(\alpha)=\frac{\sin \alpha}{\alpha},
$$

$H_{i}^{0}$ - membrane thickness at the end of the $i$-th stage $(i=1,2,3)$.

A new dimensionless time is introduced:

$$
\tau^{n}=\left(\frac{q_{0}}{H_{0}}\right)^{n} t^{n+1}
$$

As a result of a series of transformations, a connection between $\alpha$ and $\tau_{1}$ is obtained, where $\tau_{1}$ is the time of the first stage:

$$
\tau_{1}(\alpha)=\left\{(n+1) \cdot\left(\frac{2}{\sqrt{3}}\right)^{n+1} \cdot \frac{1}{k^{n}} \cdot \int_{0}^{\alpha}\left(\frac{1}{\alpha}-\operatorname{ctg} \alpha\right)\left(\frac{\sin ^{2} \alpha}{\alpha}\right)^{n} d \alpha\right\}^{\frac{1}{n}}
$$

Fig. 2 shows the dependences $\alpha\left(\tau_{1}\right)$ at $n=5, k_{1}=0.5, k_{2}=1.0$ and $k_{3}=1.5$, in this case $\alpha\left(\tau_{1}^{0}\right)=\alpha_{1}=\frac{\pi}{2}$. 


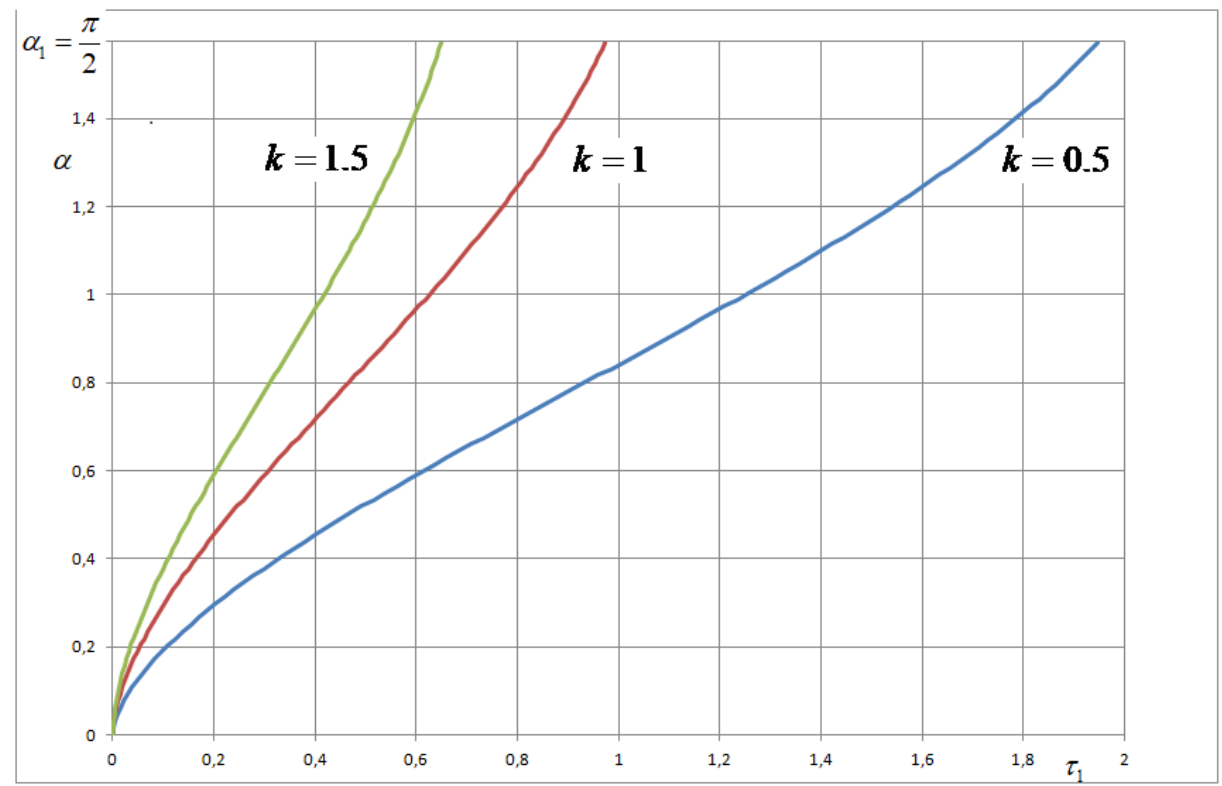

Fig. 2. Dependence of $\alpha$ on $\tau_{1}$ characterizing the first stage of membrane creep

Further, the creep of a membrane inside a rigid matrix under different contact conditions is considered.

\section{Ideal sliding of the membrane along the sides of the matrix}

\subsection{Second stage $\left(0 \leq y_{0} \leq b-1\right)$}

Introduce the coordinates of the matrix cross section $x$ and $y$ (Fig. 1) and additional dimensionless coordinates:

$$
\bar{x}=\frac{x}{a}, \bar{y}=\frac{y}{a}, \bar{b}=\frac{b}{a}, \bar{x}_{0}=\frac{x_{0}}{a}, \bar{y}_{0}=\frac{y_{0}}{a}, \bar{\rho}=\frac{\rho}{a}
$$

$\left(x_{0}, y_{0}\right.$ - coordinates of the points of contact between the membrane and the matrix), further on, the dashes above these dimensionless variables are also omitted.

In this paper, the creep of the membrane inside a relatively high matrix is considered $(b \geq 1)$.

Due to the axial symmetry of the membrane and the matrix, the creep of the right half of the membrane in coordinates $0 \leq x \leq 1,0 \leq y \leq b$ is considered below.

At $\tau>\tau_{1}^{0}$ a part of the membrane surface is adjacent to the inner longitudinal surface of the matrix.

In the study of the second stage of membrane creep, there are distinguished two close deformed states with the radius of the free arc of the membrane $\rho=1$ - one is characterized by the length of the contact area $y_{0}$, and the other is characterized by the length of the contact area $y_{0}+d y_{0}$. According to the definition of $d p_{\theta \theta}$ :

$$
d p_{\theta \theta}=\frac{\left(y_{0}+d y_{0}+0.5 \pi\right)-\left(y_{0}+0.5 \pi\right)}{y_{0}+0.5 \pi}=\frac{d y_{0}}{y_{0}+0.5 \pi}=-\frac{d H_{2}}{H_{2}},
$$

The second stage $\left(\tau_{2}=\tau_{2}^{0}\right)$ ends when the membrane touches the transverse wall of the matrix, i.e. at $y_{0}=b-1$. 
After a series of transformations, the dependence of the coordinate of the membrane contact with the matrix $y_{0}$ on time $\tau_{2}$ is obtained

$$
\tau_{2}\left(y_{0}\right)=\left\{\left(\tau_{1}^{0}\right)^{n}+\left[\frac{(0.5 \pi)^{-n}}{n}-\frac{\left(y_{0}+0.5 \pi\right)^{-n}}{n}\right]\left(\frac{2}{\sqrt{3}}\right)^{n+1} \cdot \frac{(n+1)}{k^{n}}\right\}^{\frac{1}{n}}, \quad \tau_{2}^{0}=\tau_{2}\left(y_{0}=b-1\right)
$$

\subsection{Third stage $\left(b-1 \leq y_{0} \leq b-\Delta\right)$}

At this stage, the membrane touches both sides of the matrix.

$$
d p_{\theta \theta}=\frac{(2-0.5 \pi) d y_{0}}{\left[2 y_{0}-b+1+0.5 \pi\left(b-y_{0}\right)\right]}=-\frac{d H_{3}}{H_{3}} .
$$

Stage III ends at the value $y_{0}^{0}=b-\Delta$ satisfying the inequality $\Delta=b-y_{0}^{0}<<1$.

After a series of transformations, the dependence of the coordinate $y_{0}$ on time $\tau_{3}$ is obtained:

$$
\begin{aligned}
& \tau_{3}\left(y_{0}\right)=\left\{\left(\tau_{2}^{0}\right)^{n}+(n+1) \cdot\left(\frac{2}{\sqrt{3}}\right)^{n+1} \cdot \frac{(2-0.5 \pi)}{k^{n}} \cdot \int_{(b-1)}^{y_{0}} \frac{d y_{0}}{\left[1-b+0.5 \pi b+y_{0}(2-0.5 \pi)\right]^{n+1} \cdot\left(b-y_{0}\right)^{n}}\right\}^{\frac{1}{n}}, \\
& \tau_{3}^{0}=\tau_{3}\left(y_{0}=y_{0}^{0}\right) .
\end{aligned}
$$

\section{Adhesion of the membrane along the sides of the matrix}

\subsection{Second stage $\left(0 \leq y_{0} \leq b-1\right)$}

In the study of the second stage of membrane creep, there are distinguished two close states with the radius of the free arc of the membrane $\rho=1$. According to the definition of $p_{\theta \theta}$ :

$$
\begin{array}{r}
-\int_{H_{1}^{0}}^{H_{2}\left(y_{0}\right)} \frac{d H_{2}}{H_{2}}=\int_{0}^{y_{0}} \frac{2 d y_{0}}{\pi}=\frac{2}{\pi} y_{0}=\ln \frac{H_{1}^{0}}{H_{2}\left(y_{0}\right)}, \\
H_{2}\left(y_{0}\right)=H_{1}^{0} \exp \left(-\frac{2}{\pi} y_{0}\right), \quad \dot{p}_{u}=\frac{2}{\sqrt{3}} \cdot \dot{p}_{\theta \theta}=-\frac{\dot{H}_{2}}{H_{2}}=\frac{2}{\sqrt{3}} \cdot \frac{2}{\pi} \cdot \frac{d y_{0}}{d t} .
\end{array}
$$

After a series of transformations, the dependence of the coordinate $y_{0}$ on time $\tau_{2}$ is obtained:

$$
\tau_{2}\left(y_{0}\right)=\left\{\left(\tau_{1}^{0}\right)^{n}+(n+1)\left(\frac{4}{\pi \sqrt{3}}\right)^{n+1} \cdot \frac{1}{k^{n}} \cdot \frac{\pi}{2 n} \cdot\left[1-\left(\exp \left(\frac{2}{\pi} y_{0}\right)\right)^{-n}\right]\right\}^{\frac{1}{n}}, \tau_{2}^{0}=\tau_{2}\left(y_{0}=b-1\right)
$$




\subsection{Third Stage $\left(b-1 \leq y_{0} \leq b-\Delta\right)$}

At this stage, the creep of the membrane when it touches the longitudinal and transverse sides of the matrix is described similarly:

$$
\begin{aligned}
& d p_{\theta \theta}=\frac{(2-0.5 \pi) d y_{0}}{0.5 \pi\left(b-y_{0}\right)}=-\frac{d H_{3}}{H_{3}} . \\
& H_{3}\left(y_{0}\right)=\frac{2}{\pi} \exp \left(-\frac{2}{\pi}(b-1)\right) \cdot\left(b-y_{0}\right)^{\frac{4}{\pi}-1} .
\end{aligned}
$$

Introduce the expressions for $\sigma_{u}$ and $\dot{p}_{u}$ into (1).

After a series of transformations, the dependence of the coordinate $y_{0}$ on time $\tau_{3}$ is obtained:

$$
\tau_{3}\left(y_{0}\right)=\left\{\left(\tau_{2}^{0}\right)^{n}+(n+1) \cdot\left(\frac{4}{\pi \sqrt{3}}\right)^{n+1} \frac{(2-0.5 \pi)}{k^{n}} \cdot\left\{\exp \left(\frac{2}{\pi}(b-1)\right)\right\}^{-n} \cdot \frac{\pi \cdot\left(b-y_{0}\right)^{\left(\frac{4}{\pi}-2\right) n}-\pi}{2(\pi-2) \cdot n}\right\}^{\frac{1}{n}}
$$

Stage III $\left(\tau_{3}=\tau_{3}^{0}\right)$ ends at $y_{0}^{0}$ satisfying the inequality $\Delta=b-y_{0}^{0}<<1, \tau_{3}^{0}=\tau_{3}\left(y_{0}=y_{0}^{0}\right)$.

\section{Calculation results}

It is of interest to find out under which contact condition the time of nearly complete adhesion of the membrane to the matrix is longer. This feature of the solution depends on the values of $n$ and $b$.

First, consider the results of calculating $\tau_{3}^{0}$ at $n=3$.

The results of calculations showed that in this case, for a relatively small value of $b$ (in particular, $b=3$ ) for different values of the remaining parameters ( $k$ and $\Delta$ ), the times $\tau_{3}^{0}$ in the case of ideal slip are shorter than in the case of adhesion of the membrane to the matrix. With a larger value of $b$ (in particular, $b=5$ ) the opposite result is observed. This effect is explained by the fact that, in the case of adhesion of the membrane to the matrix, the thickness of the free segment of the membrane $H_{3}\left(y_{0}\right)$ during its creep rapidly decreases in time due to an increase in the value $y_{0}$ $H_{3}\left(y_{0}\right)=\frac{2}{\pi} \exp \left(-\frac{2}{\pi}(b-1)\right) \cdot\left(b-y_{0}\right)^{\frac{4}{\pi}-1}$. Therefore, the value of the transverse creep strain $p_{\theta \theta}$ increases rather rapidly and, accordingly, the time $\tau_{3}^{0}$ decreases.

For $n=5$ the dependence $\tau_{3}^{0}$ on the type of contact conditions has a different nature - in the entire range of the parameters used, the times $\tau_{3}^{0}$ in the case of ideal slip are longer than in the case of adhesion.

For $i=1,2$, the table below provides the results of calculating the times $\tau_{1}^{0}, \tau_{2}^{0}, \tau_{3}^{0}$ corresponding to the ends of stages I, II and III of membrane deformation in the case of ideal slip of the membrane relative to the matrix at $n=5, b=1$ and $b=5$ and for $i=3,4$, it provides the values $\tau_{1}^{0}, \tau_{2}^{0}, \tau_{3}^{0}$ at the same values of $k, n, \Delta$ and $b$ in the case of adhesion of the membrane to the matrix. In Fig. 3 , the solid lines show the dependences $y_{0}\left(\tau_{3}\right)$ in the case of ideal sliding at $n=5, b=5$, 
$\Delta=0.01$, at three values of $k: k_{1}=0.5, k_{2}=1.0$ and $k_{3}=1.5$. In the same Fig. 3 , the dashed lines show the dependences $y_{0}\left(\tau_{3}\right)$ in the case of adhesion at the same values of the parameters used.

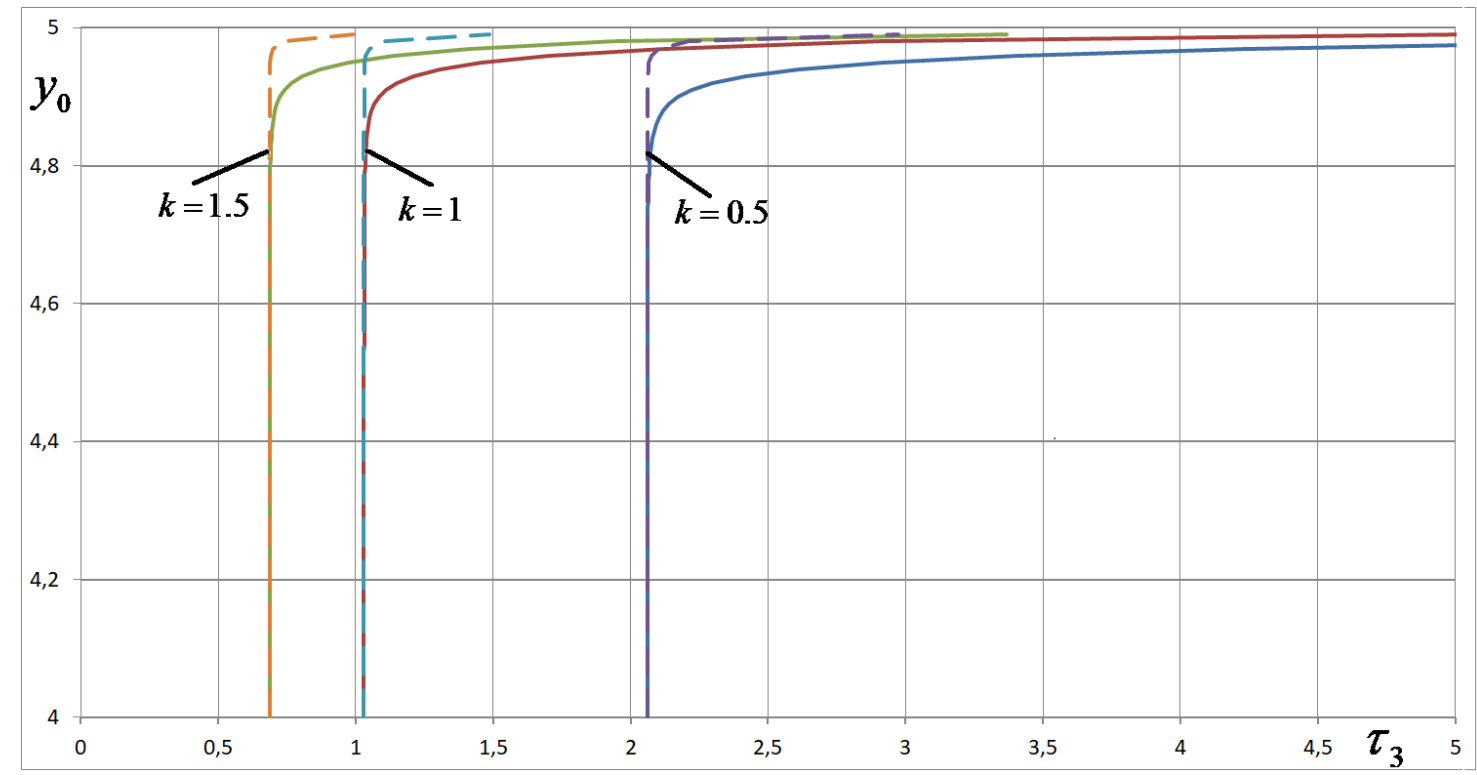

Fig. 3. Dependences of $y_{0}$ on $\tau_{3}$ in the case of ideal slip (solid lines) and in the case of adhesion (dashed lines)

Table. Values of times characterizing the end of various stages of membrane creep in the cases of ideal sliding and adhesion of the membrane relative to the matrix.

\begin{tabular}{|c|c|c|c|c|c|c|c|}
\hline$i$ & $b$ & $k$ & $\tau_{1}^{0}$ & $\tau_{2}^{0}$ & $\begin{array}{c}\Delta=0.01 \\
\tau_{3}^{0}\end{array}$ & $\begin{array}{c}\Delta=0.001 \\
\tau_{3}^{0}\end{array}$ & $\begin{array}{c}\Delta=0.0001 \\
\tau_{3}^{0}\end{array}$ \\
\hline \multirow{3}{*}{1} & \multirow{3}{*}{1} & 0.5 & 1.942 & 1.942 & 37.8 & 238.0 & 1501 \\
\hline & & 1.0 & 0.971 & 0.971 & 18.9 & 119.0 & 750 \\
\hline & & 1.5 & 0.647 & 0.647 & 12.6 & 79.3 & 500 \\
\hline \multirow{3}{*}{2} & \multirow{3}{*}{5} & 0.5 & 1.942 & 2.061 & 10.1 & 63.7 & 402 \\
\hline & & 1.0 & 0.971 & 1.030 & 5.1 & 31.8 & 201 \\
\hline & & 1.5 & 0.647 & 0.687 & 3.4 & 21.2 & 134 \\
\hline & & & & & & & \\
\hline \multirow{3}{*}{3} & \multirow{3}{*}{1} & 0.5 & 1.942 & 1.942 & 36.7 & 165.4 & 1042 \\
\hline & & 1.0 & 0.971 & 0.971 & 18.3 & 97.7 & 521 \\
\hline & & 1.5 & 0.647 & 0.647 & 12.2 & 65.1 & 347 \\
\hline \multirow{3}{*}{4} & \multirow{3}{*}{5} & 0.5 & 1.942 & 2.061 & 3 & 15.3 & 82 \\
\hline & & 1.0 & 0.971 & 1.030 & 1.5 & 7.7 & 41 \\
\hline & & 1.5 & 0.647 & 0.687 & 1.0 & 5.1 & 27 \\
\hline
\end{tabular}




\section{Conclusion}

The results of a study of the steady-state creep of a long narrow membrane inside a high rigid matrix with a proportional dependence of the transverse pressure on time are presented. Two variants of the conditions of contact between the membrane and the matrix are considered - ideal sliding and adhesion. The analysis is carried out until the time of almost complete adherence of the membrane to the matrix. These times are compared for ideal slip and adhesion for different values of the exponent in the constituting equation and the relative height of the matrix.

\section{Acknowledgements}

The authors express their gratitude to the student of the Moscow State University after M.V. Lomonosov P.M. Tretyakov for his participation in the calculations.

The work was carried out with partial financial support from the Russian Foundation for Basic Research (project code 20-08-00387).

\section{References}

[1] L. Kachanov, Fundamentals of Fracture Mechanics. Moscow: Nauka, 1974.

[2] F. Odqvist, Mathematical Theory of Creep and Creep Rupture. Second edition. Oxford at the Clarendon Press, 1974.

[3] N. Malinin, Creep in Metal Processing. Moscow: Mashinostroyeniye, 1986.

[4] A. Lokoshchenko, Creep and Long-term Strength of Metals. CISP. CRC Press. Taylor \& Francis Group. Boca. Raton. London. NewYork, 2018. 